EDITORIAL

Klin. Pädiat. 197 (1985) $181-182$

(C) F. Enke Verlag Stuttgart

\title{
Psychosoziale Betreuung krebskranker Kinder und ihrer Familien: Erfordernisse und Realität
}

\author{
G. Schellong \\ Universitäts-Kinderklinik Münster
}

Wer heute als Arzt mit krebskranken Kindern zu tun hat, kann bei seinen Gesprächen mit Eltern und Patienten von ganz anderen Voraussetzungen ausgehen als noch vor 15 Jahren. Die Diagnose einer Leukämie oder eines malignen Tumors bedeutet nicht mehr von vorneherein eine ausweglose Situation, d. h. über kurz oder lang den Tod des Kindes. Vielmehr darf bei der Mehrzahl der Patienten eine begruindete Hoffnung auf endgulltige Heilung vermittelt werden. Und doch hat das Ausmaß der seelischen Belastungen für die betroffenen Kinder und ihre Familien nicht abgenommen. Die Probleme sind im Gegenteil zahlreicher und vielgestaltiger geworden, bedingt vor allem durch die Auswirkungen der meist langen und mit vielen Nebenwirkungen und Belästigungen verbundenen Therapie. Dazu kommen die jahrelange Unsicherheit über das endgültige Schicksal des Kindes mit den Ängsten vor einem Rezidiv und die besonders schweren Probleme, die nach einem Rezidiv zu bewältigen sind.

Das schon recht umfängliche Schrifttum der letzten Zeit zur psychosozialen Betreuung krebskranker Kinder spiegelt diese Vielgestaltigkeit der Probleme wider. Die Sterbeproblematik, deren Darstellung die Literatur vergangener Jahre weitgehend beherrschte, stellt jetzt nur einen, wenn auch nach wie vor sehr wichtigen Aspekt unter vielen anderen dar. Es fehlen aber noch ausführliche Untersuchungen über Häufigkeit und relative Bedeutung der verschiedenen Problembereiche als Grundlagen fuir umfassende integrierte Versorgungskonzepte.

Hier macht eine in diesem Heft erscheinende Arbeit (1) aus der Hamburger Universitäts-Kinderklinik einen wichtigen Anfang, indem sie die dokumentierten und klassifizierten Gesprächsinhalte von über 600 Kontakten zwischen Eltern bzw. Patienten und den auf der Onkologischen Station tätigen Psychologen darstellt und diskutiert. Beeindruckend ist die große Häufigkeit der an die Psychologen herangetragenen Fragestellungen, wobei neben krankheitsund therapiebezogenen Bewältigungsproblemen (bei $52 \%$ der Familien) insbesondere Bereiche auftauchen, die zum eigentlichen Arbeitsgebiet der klinischen Psychologie gehören: Verhaltensstörungen (37\%), familiäre Probleme (33\%) und Spätfolgen (26\%).

In zwei anderen Arbeiten dieses Heftes wird ebenfalls unter Beteiligung von Psychologen über Untersuchungen auf Langzeitfolgen bei in Remission befindlichen Kindern mit Hirntumoren (2) und mit neurologischen Komplikationen in der Akutphase einer Leukämie (3) berichtet. Speziell für die Patienten mit Hirntumoren machen die Autoren darauf aufmerksam, daß bei gründlicher Untersuchung sehr viel häufiger neurologische und psychosoziale Störungen gefunden werden, als dies bei der üblichen Nachsorge zutage tritt.

Alle drei Publikationen machen sichtbar, daß in der Betreuung krebskranker Kinder psychosoziale Aspekte in großem Umfang berücksichtigt werden müssen, wie bei vielen chronischen Erkrankungen bei Kindern und Jugendlichen auch. In der Tat bemühen sich heute bereits in den meisten pädiatrisch-onkologischen Einrichtungen unseres Landes neben Ärzten und Schwestern auch Psychologen, Seelsorger, Pädagogen, Sozialpädagogen, Sozialarbeiter oder Erzieher darum, den betroffenen Kindern und ihren Familien bei der Bewältigung der zahlreichen Probleme zu helfen. Die Mitarbeit von Angehörigen derartiger Berufe auf einer pädiatrisch-onkologischen Station hat sich als geradezu unerläßlich erwiesen, da die onkologisch tätigen Ärzte und Schwestern in der Regel trotz ihrer großen Bemühungen um einen verständnisvollen und humanen Umgang mit ihren Patienten und deren Eltern meist weder genügend Zeit noch die spezielle Ausbildung besitzen, um sich in erforderlichem Umfange und mit dem benötigten fachlichen Hintergrund der psychischen, sozialen und ökonomischen Probleme annehmen zu können. Dabei ist unbedingt eine Integration des Personals fur die spezielle psychosoziale Betreuung in das onkologische GesamtTeam anzustreben, wie es z. B. in dem von Knispel et al. (1) beschriebenen Modell der Fall ist.

Zwischen den in den letzten Jahren immer deutlicher erkannten Erfordernissen und den realen Verhältnissen in den pädiatrisch-onkologischen Einrichtungen besteht allerdings noch eine erhebliche Diskrepanz, vor allem wegen der meist unzureichenden Ausstattung mit Planstellen. Eine Umfrage von U. Kaufmann (4) fur die kürzlich gegründete ,Psychosoziale Arbeitsgruppe in der Pädiatrischen Onkologie" innerhalb der GPO und der DAL ebenso wie eine durch den Autor dieses Beitrags im Auftrage des Bundesgesundheitsministeriums durchgeführte Erhebung machten diesen Mangel in bedrückender Weise sichtbar. Die spezielle psychosoziale Betreuung der krebskranken Kinder erfolgt in der Bundesrepublik ganz überwiegend durch Personal, das aus befristeten Fremdmittelstellen oder geliehenen Stellen finanziert wird oder aber für die Gesamtklinik zuständig ist und in meist nur geringen Anteilen für den onkologischen Bereich zur Verfügung steht. Politische und publizistische Aktivitäten verschiedener Elterninitiativen sowie eine Große Anfrage im Bundestag (5) haben kürzlich die personellen Unzulänglichkeiten bei der Versorgung krebskranker Kinder, speziell 
auch bei der psychosozialen Betreuung, in das Bewußtsein der Offentlichkeit gebracht.

Trotz der ungünstigen äußeren Bedingungen haben persönliche Initiative und Engagement der Beteiligten an vielen Kliniken bereits ein gewisses Maß an psychosozialer Versorgung entstehen lassen, deren Kontinuität allerdings immer wieder gefährdet ist. Für unsere beiden Fachgesellschaften GPO und DAL bleibt es im Augenblick eine vordringliche Aufgabe, Krankenhausträger und Ministerięn ebenso wie die Krankenkassen von der Notwendigkeit zu überzeugen, daß die Berücksichtigung psychosozialer Aspekte ein unerläßlicher Teil der Krankenversorgung von Kindern mit Leukämien und Tumoren ist und nur durch ein Mindestmaß an Planstellen für spezielles Personal in erforderlichem Umfange gewährleistet werden kann.

\section{Literatur}

1 Knispel, J., R. Thiel, H. Wallis: Bereiche psychosozialer Betreuung krebskranker Kinder und ihrer Familien. Auswertung eines ganzheitlichen Versorgungsmodells. Klin. Pädiat. 197 (1985) 183-187

2 Holldack, J., R. Kalff, H. Schindler, U. Rappen, W. Havers: Neurologische und psychosoziale Störungen bei Kindern mit Hirntumoren. Klin. Pädiat. 197 (1985) 188-191

3 Korinthenberg, R., F. Janssen: Zur Prognose überlebender Kinder mit neurologischen Komplikationen in der Intensivphase der Leukämiebehandlung. Klin. Pädiat. 197 (1985) 192-195

4 Kaufmann, U.: Ergebnisse einer Umfrage zur Ausstattung der Kliniken im psychosozialen Bereich (BRD, Osterreich, Schweiz) V. Tagung „Psychosoziale Versorgung in der Pädiatrischen Onkologie" 19./20. Okt. 1984, Hamburg

5 Versorgung krebskranker Kinder in der Bundesrepublik Deutschland. Antwort der Bundesregierung auf die Große Anfrage der Abgeordneten Frau Kelly und der Fraktion Die Grünen. Deutscher Bundestag, 10. Wahlperiode, Drucksache 10/2824 vom 1.2 .85

Prof. Dr. G. Schellong, Universitäts-Kinderklinik, Albert-Schweitzer-Str. 33, D-4400 Münster 